\title{
Psychological models and a simulation method to evaluate a train-rescheduling plan with passengers' dissatisfaction
}

\author{
K. Yamauchi ${ }^{1} \&$ C. Hirai ${ }^{2}$ \\ ${ }^{1}$ Human Science Division, Railway Technical Research Institute, Japan \\ ${ }^{2}$ Signalling and Transport Information Technology Division, \\ Railway Technical Research Institute, Japan
}

\begin{abstract}
We propose psychological models and a simulation method to evaluate a trainrescheduling plan from a passengers' viewpoint. Currently we do not have a refined and established evaluation method available for a train-rescheduling plan because there are too many aspects to consider such as the scale of train traffic disruption and the extent of passengers' disadvantages. In this paper, we focused on passengers' dissatisfaction as the most important aspect for commuter trains to evaluate a train-rescheduling plan. Firstly, we made a psychological model of passengers' dissatisfaction to understand its determinants. Secondly, we made a simplified model and built it into a simulation system to calculate values that can indicate passengers' dissatisfaction to evaluate a train rescheduling plan.

Keywords: passengers' dissatisfaction, psychological model, train rescheduling, web-based questionnaire, simulation.
\end{abstract}

\section{Introduction}

Passenger railway companies have a social mission to provide safe and stable transport services. They always try to revise not only their timetables to enhance the satisfaction of passengers but also their train rescheduling policies against train traffic disruptions caused by accidents.

A disruption of train traffic can cause a large effect on passengers' dissatisfaction with train transport services especially on metropolitan commuter lines. Although train dispatchers who are in charge of making a trainrescheduling plan have to make decisions in a moment, generally they do not 
have enough information and established criteria [1]. Some train dispatchers may try to restore the train traffic as soon as possible but others may try to avoid confusion of passengers at first. Both of the viewpoints are important but train dispatchers have to prioritize the viewpoints correctly.

When an accident disrupts the train traffic, passenger railway companies or train dispatchers aim to reduce passengers' dissatisfaction because they think that it is the most important factor for both passengers and railway companies themselves. They will make all efforts to reduce the dissatisfaction in practice.

In order to evaluate a train-rescheduling plan, the most straightforward way is to measure the delay. As far as the authors know, many researches for train rescheduling algorithms with optimization techniques adopt measures to minimize the delay with several variations [2-4].

There are also measures to evaluate a train-rescheduling plan up to now from passengers' viewpoints $[5,6]$. However, they have to assume that the measures can explain passengers' inconvenience enough. In another research [7], they use a measure called passengers disutility. But the measure is basically derived to evaluate static timetables, so the situation of a train traffic disruption is not considered explicitly. One of the examples used in Japan to evaluate train traffic disruption is POINT [8]. Although the measure can indicate the scale of the train traffic disruption, it does not have enough sensitivity to distinguish the difference of the effect of train rescheduling plans. Moreover, we cannot explain what the dissatisfaction is and which factor can cause the dissatisfaction quantitatively. Therefore, we need to understand the structure of passengers' dissatisfaction to evaluate a train rescheduling plan correctly.

In this paper, we analyze and make a model of passengers' dissatisfaction with a psychological approach. "Causal attribution" is one of our interests from the psychological viewpoint [9]. It is practical to calculate dissatisfaction values that involve factors controlled directly by train rescheduling operations. Therefore, we also introduce another simplified model and a simulation technique to calculate a value of passengers' dissatisfaction and make it possible to compare train-rescheduling plans.

Our approach has two steps. Initially, we made a comprehensive model that reasons passengers' dissatisfaction with train transport services [10]. In order to make the models, we focus on a railway line that runs through a metropolitan area and collect answers to questionnaires from more than several thousand passengers who use the line when the train traffic is disrupted. Secondly, we derive another simplified model to calculate a value for evaluating and comparing train-rescheduling plans.

\section{Psychological model of passengers' dissatisfaction}

\subsection{Web-based questionnaire}

We conducted web-based questionnaires two times to collect answers from passengers who experienced a train traffic disruption on a railway line in a 
metropolitan area. The main purpose of our questionnaires was to make our psychological model introduced in Section 2.4.

In order to collect answers from passengers as soon as possible after an accident happens, we adopted a web-based questionnaire method. Although there were several companies that provide such a method in Japan, we selected one of the largest service providers.

We prepared contents of the questionnaires to ask how passengers feel about the situation caused by the train traffic disruption. When an accident happened we ordered to collect passengers' answers to the service providers for our questions. We selected respondents from passengers who used trains when the accident disrupted the train traffic on a railway line at the time.

For our convenience, we call our target line "the line." The line runs through a metropolitan area and consists of several independent lines.

\subsection{Contents of the questionnaire}

We can classify the contents of our questionnaire into six categories as follows. Although they involve thirty-eight questions in total, it is not necessary for respondents to answer all questions. Because the questions are structured, a respondent would see different questions according to his/her previous answers.

\subsection{Dissatisfaction with total transport service}

We asked a passenger how he/she felt dissatisfaction with the transport service of the day in total. The main question was "How did you feel dissatisfaction with the transport service compared with the normal service?" It would appear after the passenger had answered almost all other questions. The extent of dissatisfaction was specified by five levels as follows.

(5) I felt a large amount of dissatisfaction,

(4) I felt dissatisfaction very much,

(3) I felt dissatisfaction,

(2) I felt dissatisfaction a little,

(1) I did not feel dissatisfaction at all.

We measured the level of dissatisfaction of each passenger with the values.

\subsubsection{Responsibility of railway companies}

This category involved our featured questions. "Causal attribution" was one of our interests from the psychological viewpoint. We asked a passenger about one of the reasons why he/she felt the dissatisfaction. We prepared three sentences to ask how the passenger felt as follows.

- The railway company has the main responsibility for my feeling the dissatisfaction.

- I have the main responsibility for my feeling the dissatisfaction.

- Neither the railway company nor I have the main responsibility for my feeling the dissatisfaction. 
For each sentence, we prepared the following five answer choices.

(5) Agree with it strongly,

(4) Agree with it very much,

(3) Agree with it,

(2) Agree with it a little,

(1) Disagree.

\subsubsection{Experience against expectations}

In this category, we asked how a passenger felt the difference between the disrupted transport service and the normal service with more than forty minor questions such as "It was more congested than usual in the train" and "You had to pay more to change your route." For these questions, we prepared answer choices as almost the same with those of the previous category. However, because the minor questions involve detailed subjects and we did not know how passengers remembered, we added an answer choice "I don't know."

\subsubsection{Timing of knowing the disruption}

This category involved only one question. We asked a passenger when he/she knew the train traffic was disrupted. We also wanted to know the importance of information provision to passengers.

\subsubsection{Degree of urgent}

This category also involved only one question. We asked a passenger how he/she hurried on the way to his/her destination. We prepared answer choices for four degrees of urgent.

(4) I was in a very hurry,

(3) I was in a hurry,

(2) I was in a bit of a hurry,

(1) I was not in a hurry.

\subsubsection{Individual attributes}

We also asked about general questions such as age, gender, his/her route to the destination and other individual attributes.

\subsection{Data collection}

We conducted web-based questionnaires in December 2009 and in June 2010. When an accident occurred, we immediately made a decision on whether we should conduct our survey with the train traffic disruption caused by the accident or not. Our main target was an accident that suspended train traffic along the line about an hour and that caused six to eight cancelled trains. We excluded an accident that occurred in the early morning and late at night.

Table 1 shows the overview of accidents that almost fulfilled our conditions. We could collect 7,229 answers in total. The first accident occurred in December 2009 with 90 minutes suspension of train traffic in the morning. The second occurred in June 2010 with 40 minutes' suspension of train traffic in the 
Table 1: Overview of two accidents for our research.

\begin{tabular}{ccccccc}
\hline Num. & Date & Accident & Occurrence time & Suspension & Location & $\begin{array}{c}\text { Num. of } \\
\text { answers }\end{array}$ \\
\hline \hline 1 & Dec. 2009 & Suicide & $8: 30$ a.m. & 90 min. & $\begin{array}{c}\text { Inside the city } \\
\text { area }\end{array}$ & 3,520 \\
\hline 2 & Jun. 2010 & $\begin{array}{c}\text { Signalling } \\
\text { system failure }\end{array}$ & $2: 30$ p.m. & 40 min. & $\begin{array}{c}\text { Suburban } \\
\text { area }\end{array}$ & 3,709 \\
\hline
\end{tabular}

afternoon. Because there occurred other small troubles after the 40 minutes suspension, it took much time to restore the train traffic in the second disruption.

From the viewpoint of ensuring the preciseness of respondents' memory, we selected answers that were collected within three days after the accident because old memory of humans can involve inaccuracy of the feeling at the time. We also selected answers that the respondent knew the occurrence of the accident and that they were from 20 year-old to 80 year-old. We decided to remove uncompleted answers and to analyze 5,383 selected answers. We did not separate answers by gender but they involved 3,666 of male and 1,717 of female. Moreover, we did not ask the nationality but we could guess that almost all respondents were Japanese.

\subsection{Passengers' dissatisfaction model}

We analyzed the answers to the questionnaires and made a psychological model that specifies the structure of passengers' dissatisfaction with a train traffic disruption.

We made the model by covariance structural equation modelling (SEM). In order to characterize passengers' dissatisfaction correctly, we introduce "degree of urgent," "responsibility of railway companies," "accident type" and other five factors. In process of the analysis, we eliminated answers that have a lack of focused variables. Therefore, we selected 3,393 answers to make the model. We call the model "full-model" to distinguish from another model to calculate dissatisfaction values with a simulation technique introduced in Section 3.

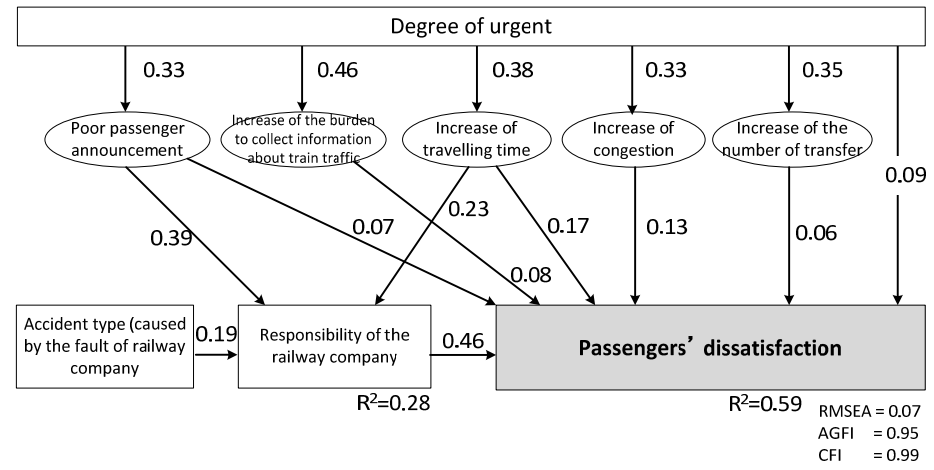

Figure 1: $\quad$ Full model of passengers' dissatisfaction. 
Figure 1 shows the full-model. The model indicates there are five (ovals) and one (rectangle) factors that dominate passengers' dissatisfaction. As mentioned in Section 2.2, passengers' dissatisfaction has measurement range of one to five.

Each of five ovals shows a factor that describes the difference from the usual transport services. Each arrow with a value shows to what extent the source factor affects the end factor. From the result of our data analysis, each of all values has one percent statistical significant level.

For example, there is an arrow from "Increase of travelling time" to "Passengers' dissatisfaction" with a value 0.17 . It means that the value of the end factor will increase 0.17 averagely when the value of the source factor increases one unit under the condition that the values of other factors are fixed. In other words, the more a value of an arrow, the more the end factor will be affected by the source factor.

\subsection{Specific feature of the full-model}

Interpreting the structure of the full-model, we can understand which factor and to what extent the factor can affect passengers' dissatisfaction as discussed in the previous section. The value of coefficient of determination of the final factor "Passengers' dissatisfaction" was 0.59 and model fit indices RMSEA [11] = $0.07, \mathrm{AGFI}=0.95$ and $\mathrm{CFI}=0.99$. Therefore, we made a decision to accept it as a model that could specify the structure of passengers' dissatisfaction.

One of the most specific features of the full-model is that the model describes not only the direct effects but also indirect effects to passengers' dissatisfaction. We assume that the structure of the dissatisfaction can involve a factor of "Cause attribution." In the full-model, the factor corresponds to the subjective evaluation for "Responsibility of the railway company." The factor and the value of the model fit indices showed that "indirect effects" can also explain passengers' dissatisfaction.

In Figure 1, we can see that there are two relations between the factor "Increase of travelling time" and "Passengers' dissatisfaction." The direct arrow with the effect value 0.17 describes a relation. We call it a direct effect. The other is described with two arrows by way of the factor of responsibility. The two arrows indicate an indirect effect. In this case, we can calculate the effect value of the indirect effect by the product of 0.23 and 0.46 . We can evaluate the effect between the factors by the summation of the direct and the indirect effects. For example, we can calculate the effect from the factor "Increase of travelling time" to "Passengers' dissatisfaction" as $0.17+0.23 * 0.46=0.28$.

Figure 2 shows the extent of each effect of five factors and a factor of "Accident type" on passengers' dissatisfaction. "Increase of travelling time" has the largest effect on passengers' dissatisfaction followed by the factor "Poor passenger announcement." we can also conclude that the effect of "Poor passenger announcement" has almost twice of the effect of "Increase of congestion." Such knowledge can support railway companies to make decisions from passengers' viewpoint. The full-model can make an important role for making policies not only for train-rescheduling but also for passenger announcements of railway companies. 


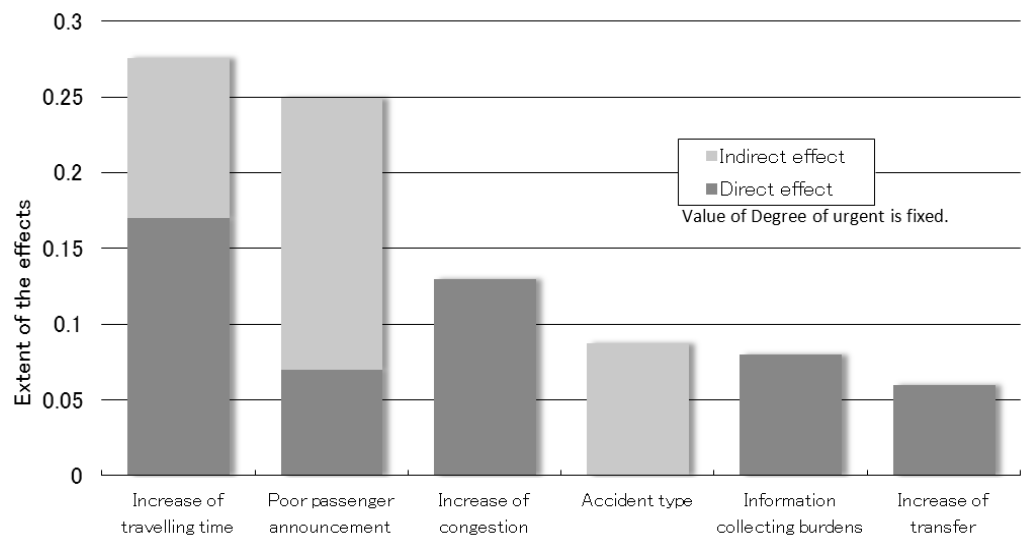

Figure 2: $\quad$ Extent of the effect on passengers' dissatisfaction.

\section{Simplified model to estimate a dissatisfaction value}

The full-model describes the structure of passengers' dissatisfaction. In this section, we focus factors that can be controlled directly by train rescheduling operations to make a model to calculate a dissatisfaction value.

At first, according to a cross validation method, we numbered the 5,383 answers described in Section 2.3 and divided them into two groups. The first group consisted of odd numbered answers and the other group consists of even numbered answers. Then we derived a model by multiple regression analysis with the first group data. The model is expressed by the following formula.

$\mathrm{PD}=-1.21+0.39 \mathrm{X}_{1}+0.20 \mathrm{X}_{2}+0.24 \mathrm{X}_{3}+0.19 \mathrm{X}_{4}$

$\mathrm{X}_{1}$ : Subjective value of increase of travelling time $(\mathrm{r}=0.55)$

$\mathrm{X}_{1}=2.69+2.46\{\log (1+\mathrm{TTD})-\log (1+\mathrm{TTN})\}+0.26 \mathrm{BTC}+0.32 \mathrm{DOU}$

$\mathrm{X}_{2}$ : Subjective value of increase of waiting time $(\mathrm{r}=0.65)$

$\mathrm{X}_{2}=2.20+1.12\{\log (1+\mathrm{WTD})-\log (1+\mathrm{WTN})\}+0.22 \mathrm{BTC}+0.32 \mathrm{BDC}+$

$0.23 \mathrm{DOU}$

$\mathrm{X}_{3}$ : Subjective value of increase of congestion rate in rolling stock $(\mathrm{r}=0.62)$

$\mathrm{X}_{3}=2.37+0.02\{\log (1+\mathrm{CRD})-\log (1+\mathrm{CRN})\}+0.27 \mathrm{BTC}+0.28 \mathrm{DOU}$

$\mathrm{X}_{4}$ : Subjective value of increase of the number of transferring $(\mathrm{r}=0.79)$

$\mathrm{X}_{4}=1.89+1.44\{\log (1+\mathrm{NTD})-\log (1+\mathrm{NTN})\}+0.34 \mathrm{BTC}+0.14 \mathrm{DOU}$

Meanings of terms described in the formula are as follows. We can calculate each term for each passenger by a simulation method shown in Section 4.

With the formula, we estimated a passenger's dissatisfaction value for each answer belonging to the even numbered group. We compared the estimated values and the corresponding answered values of passengers' dissatisfaction. As a result, the value of coefficient of determination of the dependent variable PD was 0.37 . We concluded that the formula can statically explain $37 \%$ of the variance of passengers' dissatisfaction. 
PD: Value of a passengers' dissatisfaction when the train traffic is disrupted. It has a range of one through five. The value corresponds to the score introduced for the questionnaire in Section 2.2.

TTD: Travelling time of a passenger when the train traffic is disrupted. TTD includes WTD. The value is specified in minutes.

TTN: Travelling time of a passenger when trains are operated normally. TTN includes WTN. The value is specified in minutes.

WTD: Waiting time of a passenger at stations when the train traffic is disrupted. It includes waiting time to transfer another train. The value is specified in minutes.

WTN: Waiting time of a passenger at stations when trains are operated normally. It includes waiting time to transfer another train. The value is specified in minutes.

CRD: Congestion rate value when the train traffic is disrupted. The value is specified by percentage value. If the rate is $150 \%$, the value is 150 .

CRN: Congestion rate value when trains are operated normally. The value is specified by percentage value. If the rate is $150 \%$, the value is 150 .

NTD: Number of transfer of a passenger when the train traffic is disrupted.

NTN: Number of transfer of a passenger when trains are operated normally.

BTC: Binary indicator value. When a passenger finds a train that he/she is going to get on is cancelled, it takes 1 . Otherwise, it takes 0 .

BDC: Binary indicator value. When a passenger finds the destination of a train that he/she is onboard is changed, it takes 1 . Otherwise, it takes 0 .

DOU: Value of how a passenger is in a hurry. The value corresponds to the degree of urgent introduced for the questionnaire in Section 2.2.

\section{Simulation method}

We introduce a micro-simulation technique to calculate values of passengers' dissatisfaction with the formula shown in the previous section. The simulation system [7] can calculate the value for each of passengers generated in the simulation system. We can summarize the values for all passengers in the simulation model and compare train rescheduling plans.

Basic input data to the simulation system is as follows.

(a) Timetable data of a train rescheduling plan

(b) Original timetable data

(c) Other data such as Origin-Destination (OD) data and infrastructure data

Figure 3 shows a flow of the calculation. The system calculates each passenger's experience according to the given timetable data and records how long each passenger takes to arrive at the destination station and how congested the train he/she takes is.

With the simulated records of each passenger and the formula introduced in Section 3, the system can calculate the value of dissatisfaction for the train rescheduling plan. 


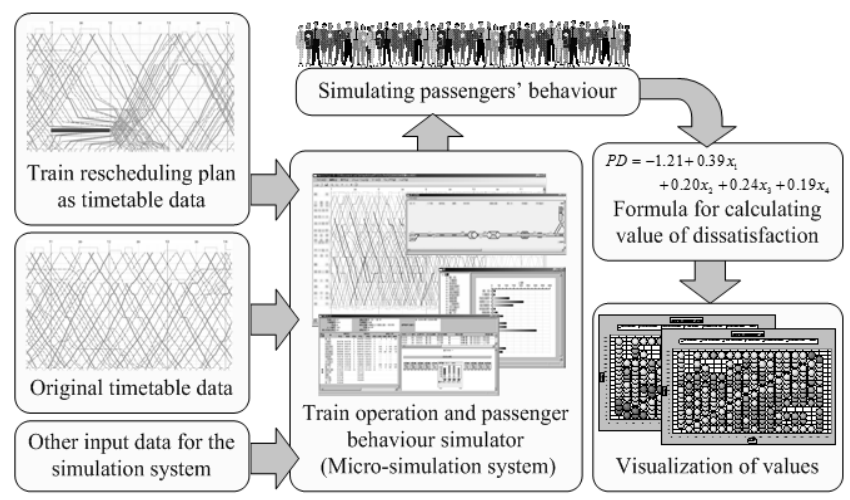

Figure 3: Flow of calculating passengers' dissatisfaction.

\section{Numerical experiment}

\subsection{Data preparation}

In order to conduct our numerical experiment, we prepared timetable data and a scenario of a train traffic disruption. Although we focused a line consists of several independent lines that runs in a metropolitan area for our questionnaires, we prepared the data for only an independent line in our numerical experiment. Limited data can allow us to investigate the values carefully.

Figure 4 shows a part of the original train diagram of the line. The horizontal axis shows time and vertical axis shows locations of stations. There are 18 stations between Stations $\mathrm{X}$ and $\mathrm{Y}$. The distance between the two stations is about $80 \mathrm{~km}$. Station $\mathrm{X}$ locates at the city side and Station Y locates at the

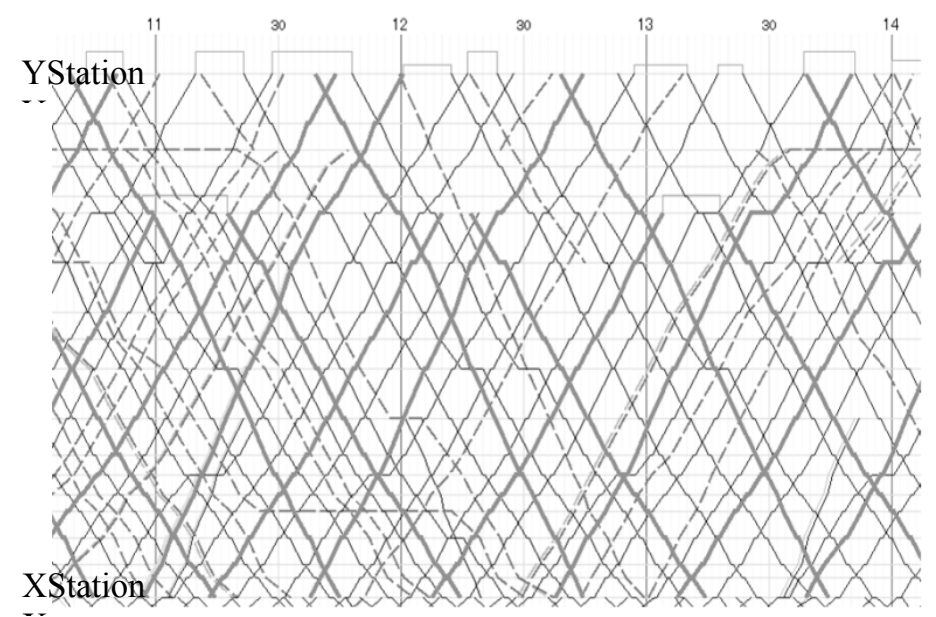

Figure 4: $\quad$ Original train diagram. 
country side. There are local trains (thin lines), freight trains (dashed lines) and long-distance trains that run through the metropolitan area (thick lines). The long-distance trains run through Station $\mathrm{X}$ and go down to the mid of the city.

The simulation system can simulate behaviours of each passenger with basic passenger data called OD data. The data is originally collected by automatic ticket inspector gates at each station. We adjusted the data with another observed data of the number of passengers onboard and converted the data to fit the input format of the simulation system.

\subsection{Scenario description}

We prepare a scenario of a train traffic disruption on the target line. The scenario is imaginary one but we referred train traffic disruptions that occurred on the line and consulted experts who had experience of train rescheduling operations.

Figure 5 shows a train diagram disrupted when an accident occurs at Station $\mathrm{Z}$ and suspends train traffic for an hour. The suspension causes a large train interval on the line even after an hour. The lack of the transport capacity will cause passengers' dissatisfaction.

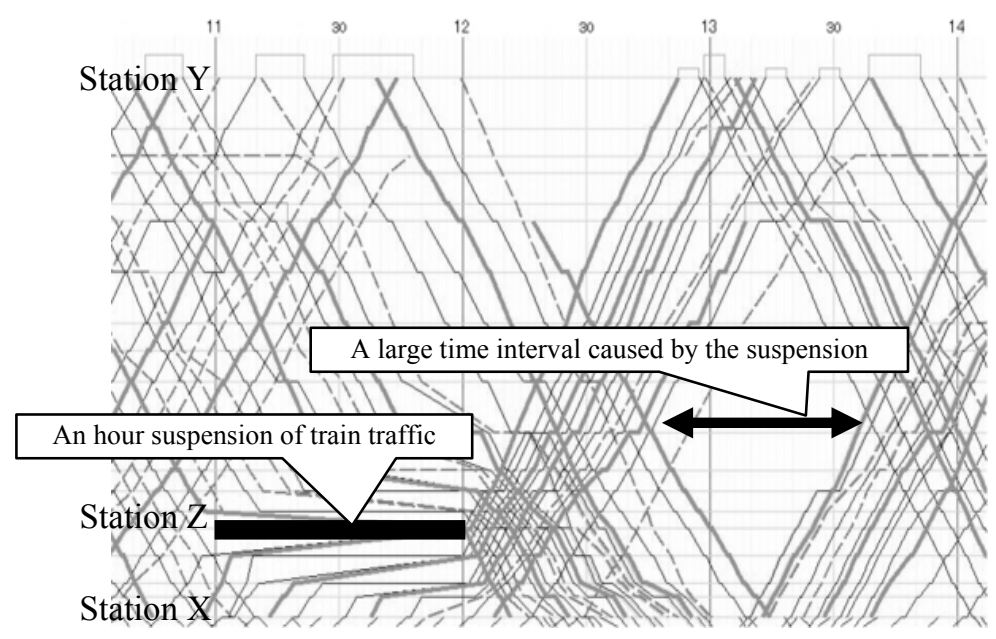

Figure 5: Train diagram with an accident.

In reality, train dispatchers will set extra trains as shown in Figure 6. We will compare the two timetable data by calculating values of passengers' dissatisfaction with the formula introduced in Section 3.

\subsection{Calculation results}

Since the simulation system can calculate the value of dissatisfaction for each simulated passenger, we can visualize as in Figures 7 and 8 . The values are summarized according to the Origin-Destination stations. Figures 7 and 8 
correspond to the train diagrams in Figures 5 and 6, respectively. We can also perceive which passengers' dissatisfaction can be reduced by the trainrescheduling operations.

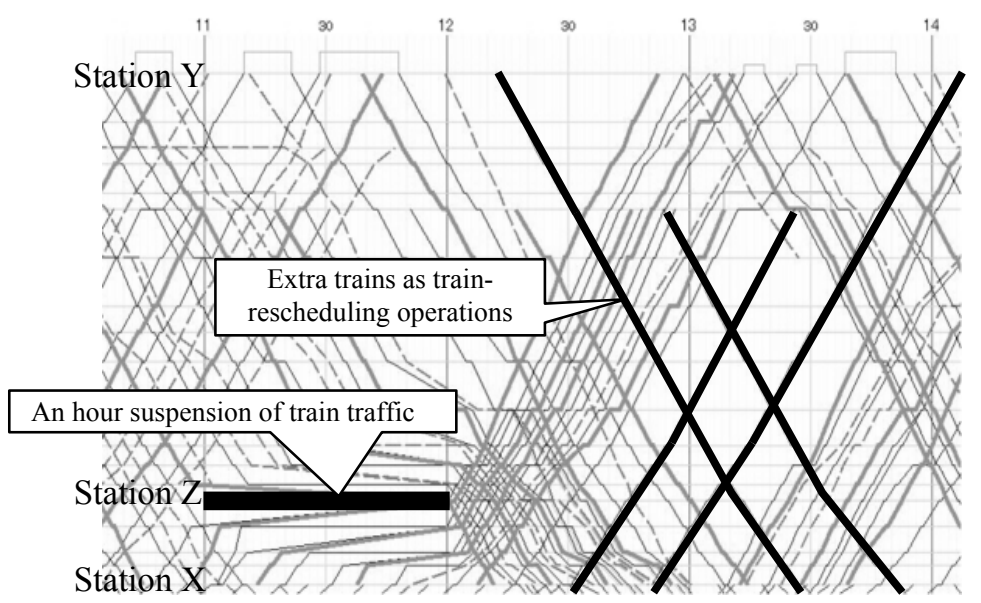

Figure 6: Train diagram with train rescheduling operations.

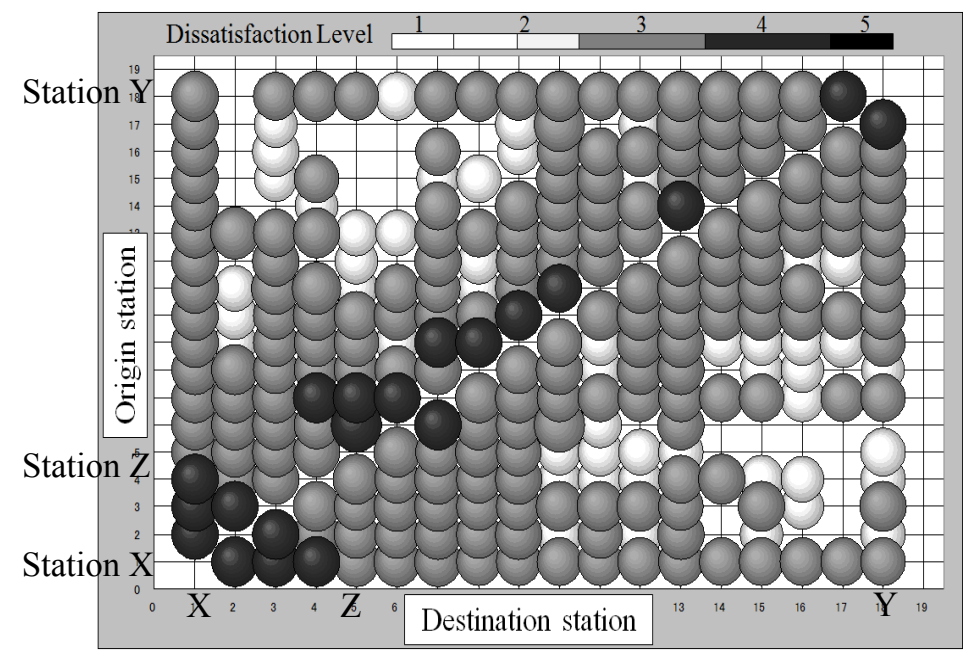

Figure 7: Visualization of the values (rescheduled). 


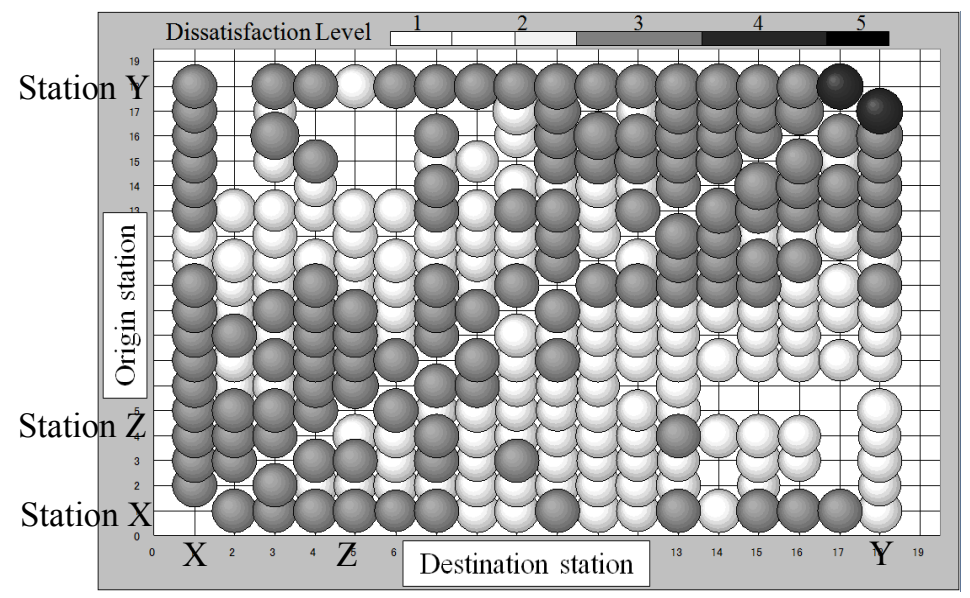

Figure 8: Visualization of the values (rescheduled).

\section{Conclusion}

We have analyzed answers to the questionnaires and derived psychological models that specify passengers' dissatisfaction with disrupted train transport services. The full-model can explain the structure of the dissatisfaction and the simplified model allows us to estimate the values of the dissatisfaction with a simulation technique. We expect that the models can support railway companies to review their train rescheduling policies against train traffic disruptions.

\section{Acknowledgement}

We appreciate our research partner $R \& D$ Centre of JR East Group (East Japan Railway Company) for their useful suggestions and providing us with data for our numerical experiments.

\section{References}

[1] Tomii, N. (ed.), Train rescheduling techniques for more punctual and stable railway transport (in Japanese), Ohmsha Publishing, Tokyo, 2010.

[2] Cordeau, J. F., Toth, P., Vigo, D., A survey of optimization models for train routing and scheduling, Transportation Science, 32(4), pp. 380-404, 1998.

[3] Törnquist, J., Railway traffic disturbance management - An experimental analysis of disturbance complexity, management objectives and limitations in planning horizon, Transportation Research Part A, 41, pp. 249-266, 2007. 
[4] Corman, F., D’Ariano, A., Pacciarelli, D., Pranzo, M., Optimal inter-area coordination of train rescheduling decisions, Transportation Research Part E, 48, pp. 71-78, 2012.

[5] Tomii, N., Tashiro, Y., Tanabe, N., Hirai, C., Muraki, K., Train rescheduling algorithm which minimizes passengers' dissatisfaction, Proc. 18th Int. Conf. on Industrial and Engineering Applications of Artificial Intelligence and Expert Systems (IEA/AIE 2005), LNCS 3533, Springer, pp. 829-838, 2005.

[6] Chigusa, K., Sato, K., Koseki, T., Passenger-oriented optimization for train rescheduling on the basis of mixed integer programming (in Japanese), IEEJ Transactions on Industry Applications, 132(2), pp. 170-177, 2011.

[7] Kunimatsu, T., Hirai, C., Tomii, N., Evaluation of timetables by estimating passengers' personal disutility using micro-simulation, Proceedings of the 3rd International Seminar on Railway Operations Modelling and Analysis (RailZurich 2009), Zurich, 2009.

[8] Tachi, M., Fukuyama, H., Development of Stability index of rail transport POINT and its extension in a railway company (in Japanese), Proc. of the 44th symposium on the use of cybernetics on the railways, 101, 2007.

[9] Weinger, B., An attributed theory of motivation and emotion, SpringerVerlag, New York, 1986.

[10] Yamauchi, K., Determinants of passengers' dissatisfaction regarding disrupted train service, The Japanese Journal of Psychology, 83, pp. 43-51, 2012.

[11] Browne, M. W. and Cudeck, R., Alternative ways of assessing model fit. In K. A. Bollen and J.S. Long (Eds.), Testing Structural Equation Model, Newbury Park CA, Sage Publications, pp. 136-162, 1993. 